\title{
Association between high-sensitivity C-reactive protein levels and clinical outcomes in acute ischemic stroke patients treated with endovascular therapy
}

\author{
Luling Wang ${ }^{1 \#}$, Longfei Wu ${ }^{1 \#}$, Ye Lang ${ }^{2}, \mathrm{Di}_{\mathrm{Wu}}{ }^{1}$, Jian $\mathrm{Chen}^{3}$, Wenbo Zhao ${ }^{1}$, Chuanhui $\mathrm{Li}^{4}, \mathrm{Xunming} \mathrm{Ji}^{3} \wedge$ \\ ${ }^{1}$ Department of Neurology and China-America Institute of Neuroscience, Xuanwu Hospital, Capital Medical University, Beijing, China; \\ ${ }^{2}$ Department of Neurology, Shengli Oilfield Central Hospital, Dongying, China; ${ }^{3}$ Department of Neurosurgery, Xuanwu Hospital, Capital Medical \\ University, Beijing, China; ${ }^{4}$ Department of Emergency, Xuanwu Hospital, Capital Medical University, Beijing, China \\ Contributions: (I) Conception and design: L Wang, L Wu, X Ji; (II) Administrative support: X Ji; (III) Provision of study materials or patients: L Wu, \\ W Zhao; (IV) Collection and assembly of data: L Wang, Y Lang, L Wu, D Wu, J Chen, W Zhao; (V) Data analysis and interpretation: L Wang, C \\ Li; (VI) Manuscript writing: All authors; (VII) Final approval of manuscript: All authors. \\ \#These authors contributed equally to this work. \\ Correspondence to: Xunming Ji. Department of Neurosurgery, Xuanwu Hospital, Capital Medical University, Beijing, China. Email: jixm@ccmu.edu.cn.
}

\begin{abstract}
Background: Increasing evidence demonstrates that high-sensitivity C-reactive protein (hs-CRP) is an independent prognostic predictor in acute ischemic stroke (AIS) patients. The purpose of this study is to investigate the association between hs-CRP levels and clinical outcomes in AIS patients receiving endovascular therapy (EVT).
\end{abstract}

Methods: This observational study was based on a prospective registry study. AIS patients receiving EVT from December 2012 to January 2019 were included. The modified Rankin Scale (mRS) scores at the 90-day and long-term follow-up were evaluated as clinical outcomes. Multivariable logistic regression analysis was conducted to adjust for confounders. Receiver operating characteristic (ROC) curve analysis was performed based on significant predictors of favorable outcomes in the logistic regression analysis. Patients were divided into two groups according to the cutoff value. Clinical outcomes were compared between groups. Survival probability was assessed using Kaplan-Meier survival analysis.

Results: Multivariable logistic regression analysis of the 362 enrolled AIS patients demonstrated that age $(\mathrm{P}=0.030)$, National Institutes of Health Stroke Scale (NIHSS) score $(\mathrm{P}=0.023)$, hs-CRP levels $(\mathrm{P}<0.001)$, and symptomatic intracranial hemorrhage $(\mathrm{sICH})(\mathrm{P}=0.006)$ were independently predictive of favorable outcomes. ROC curve analysis indicated that the hs-CRP level was predictive of favorable outcomes at the 90-day follow-up with a cutoff value of $8.255 \mathrm{mg} / \mathrm{L}$. The $\mathrm{mRS}$ scores between patients with hs-CRP $<8.255 \mathrm{mg} / \mathrm{L}$ and patients with hs-CRP $\geq 8.255 \mathrm{mg} / \mathrm{L}$ at the 90 -day [2 (IQR, 1-2) vs. 4 (IQR, 3-6), $\mathrm{P}<0.001$ ] and long-term follow-up [1 (IQR, 0-2) vs. 4 (IQR, 2-6), $\mathrm{P}<0.001]$ were significantly different. Patients with hs-CRP $\geq 8.255 \mathrm{mg} / \mathrm{L}$ had significantly increased risk of poor clinical outcomes at the 90-day and long-term follow-up compared with those with hs-CRP $<8.255 \mathrm{mg} / \mathrm{L}$ ( $\mathrm{P}<0.001$ each).

Conclusions: Elevated hs-CRP levels were associated with poor clinical outcomes in AIS patients receiving EVT.

Keywords: Ischemic stroke; endovascular therapy (EVT); high-sensitivity C-reactive protein (hs-CRP); outcome

Submitted May 09, 2020. Accepted for publication Sep 04, 2020.

doi: $10.21037 / \mathrm{atm}-20-3820$

View this article at: http://dx.doi.org/10.21037/atm-20-3820

\footnotetext{
^ Luling Wang, ORCID: 0000-0002-9113-9379; Xunming Ji, ORCID: 0000-0002-0527-2852.
} 


\section{Introduction}

Acute ischemic stroke (AIS), a serious epidemic issue, is the second leading cause of death and long-term disability worldwide $(1,2)$, and it places a tremendous burden on both healthcare systems and socioeconomic development (3). Endovascular therapy (EVT) is one of the established treatments for eligible AIS patients (4). With successful recanalization, most AIS patients with large vessel occlusion (LVO) can obtain benefits $(5,6)$. Prior studies in AIS patients with EVT have identified patient-specific factors and procedural considerations as potential predictors of poor outcome or death (7-9). However, the simple and high-sensitivity hematological indexes leading to functional dependence remain unknown.

Inflammation plays an important role in the pathogenesis of AIS (10). Previous research has suggested that cerebral ischemia triggers inflammatory responses via the upregulation of acute-phase proteins such as C-reactive protein (CRP) (11). CRP, a nonspecific biomarker of inflammation, has been proposed to be an indicator of atherosclerosis and a predictor of both cardiovascular and cerebrovascular endpoints (12). A high-sensitivity CRP (hs-CRP) assay quantifies smaller incremental changes in inflammation and is more clinically relevant than CRP for evaluating the relationship between acute inflammation and prediction of the degree of long-term disability (13).

Although increasing evidence has demonstrated that hsCRP is an independent predictor of prognosis in patients with AIS (14-18), the association between hs-CRP levels and clinical outcomes of AIS patients with EVT has not been established. Therefore, we performed this study to investigate the association between hs-CRP levels and clinical outcomes in AIS patients treated with EVT. We present the following article in accordance with the Strengthening the Reporting of Observational Studies in Epidemiology (STROBE) reporting checklist (19) (available at http://dx.doi.org/10.21037/atm-20-3820).

\section{Methods}

\section{Study design}

This study was based on a prospective registry study in Xuanwu Hospital, Capital Medical University in Beijing, China, and it was conducted in accordance with the Declaration of Helsinki (as revised in 2013). All consecutive AIS patients receiving EVT in our center were registered in this prospectively collected registry, which was approved by the Ethics Committee of Xuanwu Hospital (No. 2017030), and informed consent was obtained from all the patients.

\section{Inclusion and exclusion criteria}

AIS patients with LVO were treated with EVT following the guidelines as soon as possible after symptom onset (4). For the present study, we enrolled AIS patients treated with EVT from December 2012 to January 2019. The inclusion criteria of this study were as follows: (I) diagnosis of AIS and (II) LVO in the anterior circulation confirmed by computed tomographic angiography, magnetic resonance angiography or digital subtraction angiography. The exclusion criteria were posterior circulation strokes and loss to follow-up.

\section{$E V T$}

Based on the regulations of our center, only neurointerventionists who were trained in the technique of performing extracranial and intracranial stents were qualified to perform EVT. All procedures were performed under local or general anesthesia. Digital subtraction angiography was performed to determine the occlusion sites. Other specific intervention strategies, the type of stent retrievers, and other devices were chosen at the discretion of the treating neurointerventionist. Heparin was administered intravenously to maintain the activated coagulation time between 250 and $300 \mathrm{~s}$ during the procedure unless intravenous thrombolysis was implemented. After EVT, all patients were transferred to the neurological intensive care unit or acute stroke unit for further treatment.

\section{Clinical assessment and hs-CRP}

Baseline information, including demographics, comorbidities, pathogenesis of stroke, National Institutes of Health Stroke Scale (NIHSS) score, baseline Alberta Stroke Program Early Computed Tomography Score (ASPECTS) on computed tomography (CT) scan, vital signs, time intervals, occlusion sites, interventional procedures, and recanalization conditions, was recorded from the database. Biochemical variables and other indicators were measured within 24 hours of admission in a certified central laboratory. Stroke severity on admission was assessed by certified evaluators using the NIHSS score (20). The NIHSS score is a neurologic examination stroke scale with a total score of 42 ; a higher NIHSS score indicates a more severe disability (20). ASPECTS is a quantitative CT score with a total of score 


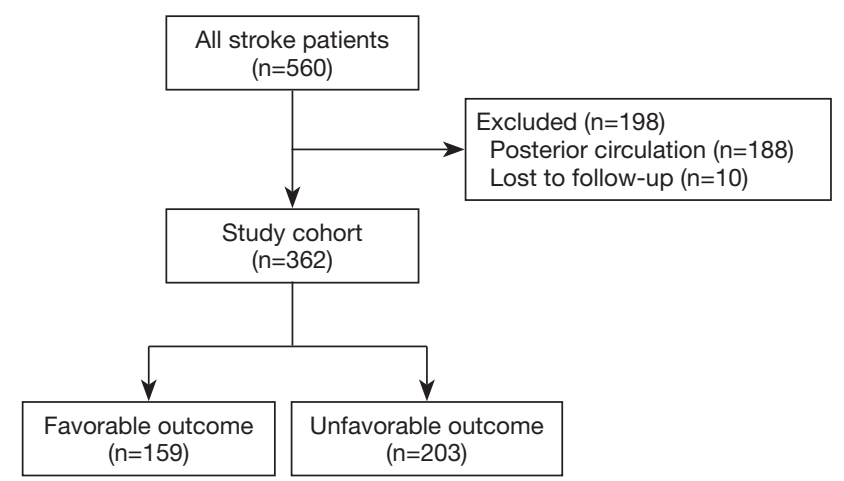

Figure 1 Flowchart of patients included in this study.

of 10. A lower ASPECTS indicates a more severe ischemic change (21).

Hs-CRP levels were measured as part of routine laboratory testing for patients with AIS on admission. HsCRP assays were performed using the immunoturbidimetric assay. We conducted a laboratory calibration study to evaluate possible differences in hs-CRP measurements between laboratories, specimen types, assay methods, instruments, and time of measurement and found that differences in hs-CRP were not large enough to warrant calibration.

\section{Outcome assessment}

Clinical outcomes were evaluated using the modified Rankin Scale (mRS) score. The mRS score is based on a 7-point scale, ranging from 0 (no symptoms) to 6 (death) (22). Clinical outcomes included the rates of favorable outcome (mRS, $0-2$ ), no or minimal disability (mRS, $0-1$ ), and mortality (mRS, 6) at 90 days as well as during long-term follow-up. In this study, an mRS score of 0-2 indicated a favorable outcome, while an mRS score of 3-6 indicated an unfavorable outcome. Outcome assessments were performed via telephone or in-person interviews by the independent evaluators who were unaware of the clinical information.

\section{Statistical analysis}

Baseline characteristics were described. Descriptive statistics are presented as the means [standard deviation (SD)] for normally distributed continuous variables, medians [interquartile range (IQR)] for nonnormally distributed continuous variables, and percentages for categorical variables. The Kolmogorov-Smirnov test was performed for the normality assessment of continuous variables. These variables were compared using the Student's $t$-test or the Mann-Whitney $U$ test for continuous variables and the chisquare test or Fisher's exact test for categorical variables.

To minimize the potential bias and adjust for confounders, a multivariable logistic regression analysis was conducted with favorable outcome at 90 days as the dependent variable and with age, male sex, NIHSS score, ASPECTS, atrial fibrillation, hs-CRP levels, time from stroke onset to recanalization, recanalization condition, and symptomatic intracranial hemorrhage (sICH) as the covariates. Additionally, receiver operating characteristic (ROC) curve analysis was performed based on the significant predictors of favorable outcome in the logistic regression analysis. The area under the curve (AUC) and Youden's index were further calculated to determine the cutoff value. Then, patients were divided into two groups according to the cutoff value, and the clinical outcomes at the 90-day and long-term followup were compared between the two groups. Furthermore, Kaplan-Meier survival analysis was performed to compare the survival probability at long-term follow-up using the logrank test.

The significance level was set at $\mathrm{P}$ value $\leq 0.05$ (two-sided). Statistical analyses were performed using SPSS 26.0 (IBM Corp., USA).

\section{Results}

\section{Demographic characteristics (Figure 1 and Table 1)}

Of the 560 patients who underwent EVT from December 2012 to January 2019 in our institution, 198 patients were excluded (188 patients had posterior circulation strokes, and 10 patients were lost to follow-up). Thus, a total of 362 patients were included for further analysis (Figure 1).

The mean age was 63.2 (SD, 12.7) years, and 249 patients $(69.8 \%)$ were male. The median NIHSS score was 14 (IQR, 11-18), and the median ASPECTS score was 8 (IQR, 8-10) at baseline. One hundred thirty-three patients $(36.7 \%)$ received intravenous alteplase before EVT. The median times from stroke onset to groin puncture (OTP), groin puncture to recanalization (PTR), and stroke onset to recanalization (OTR) were 397 (IQR, 284-519) $\mathrm{min}$, 82 (IQR, 52-120) min, and 489 (IQR, 374-609) min, respectively. A total of 210 patients (58\%) had middle cerebral artery occlusion, and 38 patients $(10.5 \%)$ suffered sICH post procedure. The median hs-CRP value of all enrolled patients was 9.15 (IQR, 3.66-16.07) mg/L. Details of the baseline characteristics are presented in Table 1. 
Table 1 Baseline characteristics

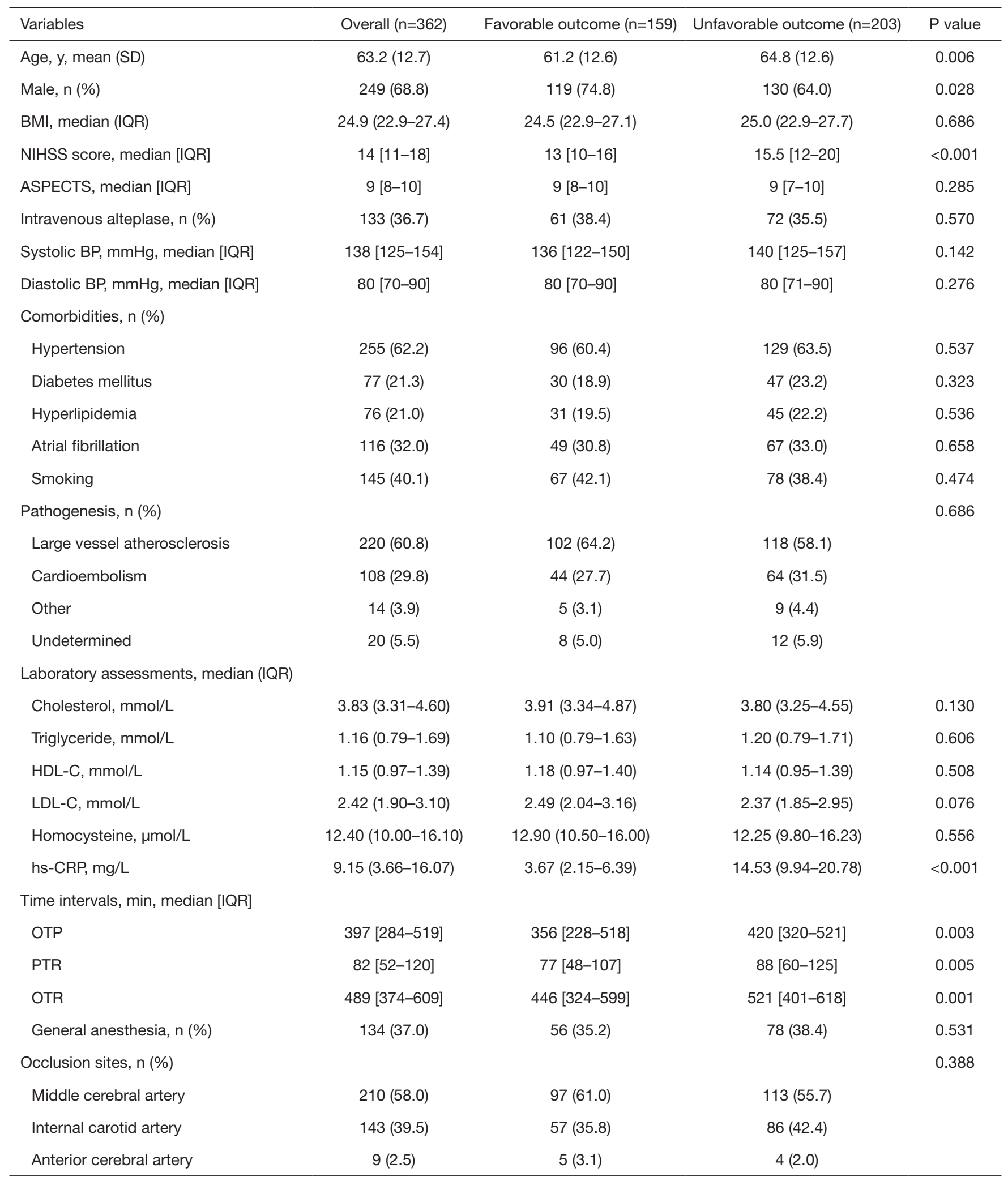

Table 1 (Continued) 
Table 1 (Continued)

\begin{tabular}{|c|c|c|c|c|}
\hline Variables & Overall $(n=362)$ & Favorable outcome $(n=159)$ & Unfavorable outcome $(n=203)$ & $P$ value \\
\hline Stent retriever & $298(82.3)$ & $127(79.9)$ & $171(84.2)$ & 0.280 \\
\hline Aspiration & $129(35.6)$ & $50(31.4)$ & $79(38.9)$ & 0.229 \\
\hline Recanalization & $304(84.0)$ & $143(89.9)$ & $161(79.3)$ & 0.006 \\
\hline
\end{tabular}

Data are expressed as the means, medians or $\mathrm{n}(\%)$. SD, standard deviation; BMI, body mass index; NIHSS, National Institutes of Health Stroke Scale; IQR, interquartile range; ASPECTS, Alberta Stroke Program Early Computed Tomography Score; BP, blood pressure; HDL-C, high-density lipoprotein cholesterol; LDL-C, low-density lipoprotein cholesterol; hs-CRP, high-sensitivity C-reactive protein; OTP, stroke onset to groin puncture; PTR, groin puncture to recanalization; OTR, stroke onset to recanalization; sICH, symptomatic intracranial hemorrhage.

Table 2 Predictors of favorable outcome at 90-day follow-up (multivariable analysis)

\begin{tabular}{lccc}
\hline Variables & OR & $95 \% \mathrm{Cl}$ & P value \\
\hline Age & 0.972 & $0.947-0.977$ & 0.030 \\
Male & 1.752 & $0.897-3.420$ & 0.100 \\
NIHSS score & 0.941 & $0.892-0.991$ & 0.023 \\
ASPECTS & 1.044 & $0.842-1.295$ & 0.694 \\
Atrial fibrillation & 1.792 & $0.876-3.666$ & 0.110 \\
hs-CRP & 0.858 & $0.817-0.900$ & $<0.001$ \\
OTR & 1.000 & $0.998-1.001$ & 0.752 \\
Recanalization & 2.083 & $0.744-5.826$ & 0.162 \\
sICH & 0.143 & $0.036-0.570$ & 0.006 \\
\hline
\end{tabular}

OR, odds ratio; $\mathrm{CI}$, confidence interval; NIHSS, National Institutes of Health Stroke Scale; ASPECTS, Alberta Stroke Program Early Computed Tomography Score; hs-CRP, high-sensitivity $\mathrm{C}$-reactive protein; OTR, stroke onset to recanalization; sICH, symptomatic intracranial hemorrhage.

\section{Outcomes}

In the multivariable logistic regression analysis, after adjusting for confounding factors, age [odds ratio (OR), 0.972; 95\% confidence interval (CI), 0.947-0.977; $\mathrm{P}=0.030$ ], NIHSS score (OR, 0.941; 95\% CI, 0.892-0.991; $\mathrm{P}=0.023$ ), hs-CRP levels (OR, 0.858; 95\% CI, 0.817-0.900; $\mathrm{P}<0.001$ ), and $\mathrm{sICH}(\mathrm{OR}, 0.143 ; 95 \% \mathrm{CI}, 0.036-0.570 ; \mathrm{P}=0.006)$ were significantly associated with favorable outcomes at 90 days post-EVT (Table 2).

The ROC curve analysis indicated that the hs-CRP level was a predictor of favorable outcome at the 90-day follow-

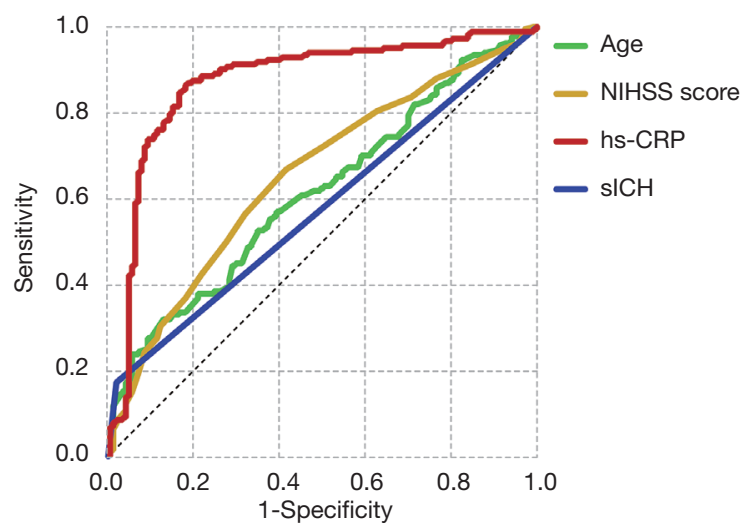

Figure 2 ROC curves of predictors of favorable outcome at 90-day follow-up. ROC, receiver operating characteristic; NIHSS, National Institutes of Health Stroke Scale; hs-CRP, high-sensitivity C-reactive protein; sICH, symptomatic intracranial hemorrhage.

up with an AUC of 0.871 and a cutoff value of $8.255 \mathrm{mg} / \mathrm{L}$ (sensitivity, $86.7 \%$; specificity, $83 \%$; Figure 2).

Patients were then divided into two groups based on the cutoff value $(8.255 \mathrm{mg} / \mathrm{L})$. The median 90 -day mRS scores were 2 (IQR, 1-2) in patients with hs-CRP $<8.255 \mathrm{mg} / \mathrm{L}$ and 4 (IQR, 3-6) in patients with hs-CRP $\geq 8.255 \mathrm{mg} / \mathrm{L}(\mathrm{P}<0.001)$. At long-term follow-up (median follow-up time, 15 months), the median mRS scores were 1 (IQR, 0-2) and 4 (IQR, 2-6) in patients with hs-CRP $<8.255$ and $\geq 8.255 \mathrm{mg} / \mathrm{L}$, respectively $(\mathrm{P}<0.001)$. The rates of favorable outcome, no or minimal disability, and mortality were significantly different between the two groups at 90-day and long-term follow-up $(\mathrm{P}<0.001$ each). The details of functional outcomes and the distribution of 
Table 3 Functional outcomes at 90-day and long-term follow-up

\begin{tabular}{|c|c|c|c|c|}
\hline Variables & Overall $(n=362)$ & hs-CRP <8.255 mg/L (n=159) & hs-CRP $\geq 8.255$ mg/L $(n=203)$ & $P$ value \\
\hline mRS, median [IQR] & 3 [2-5] & $2[1-2]$ & $4[3-6]$ & $<0.001$ \\
\hline Favorable outcome, n (\%) & $159(43.9)$ & $132(83.0)$ & $27(13.3)$ & $<0.001$ \\
\hline No or minimal disability, $\mathrm{n}(\%)$ & $77(21.3)$ & $71(44.7)$ & $6(3.0)$ & $<0.001$ \\
\hline \multicolumn{5}{|l|}{ Long-term follow-up } \\
\hline mRS, median [IQR] & $2[1-5]$ & $1[0-2]$ & $4[2-6]$ & $<0.001$ \\
\hline Favorable outcome, n (\%) & $189(52.2)$ & $134(84.3)$ & $55(27.1)$ & $<0.001$ \\
\hline No or minimal disability, $\mathrm{n}(\%)$ & $119(32.9)$ & $93(58.5)$ & $26(12.8)$ & $<0.001$ \\
\hline
\end{tabular}

hs-CRP, high-sensitivity C-reactive protein; mRS, modified Rankin Scale; IQR, interquartile range.

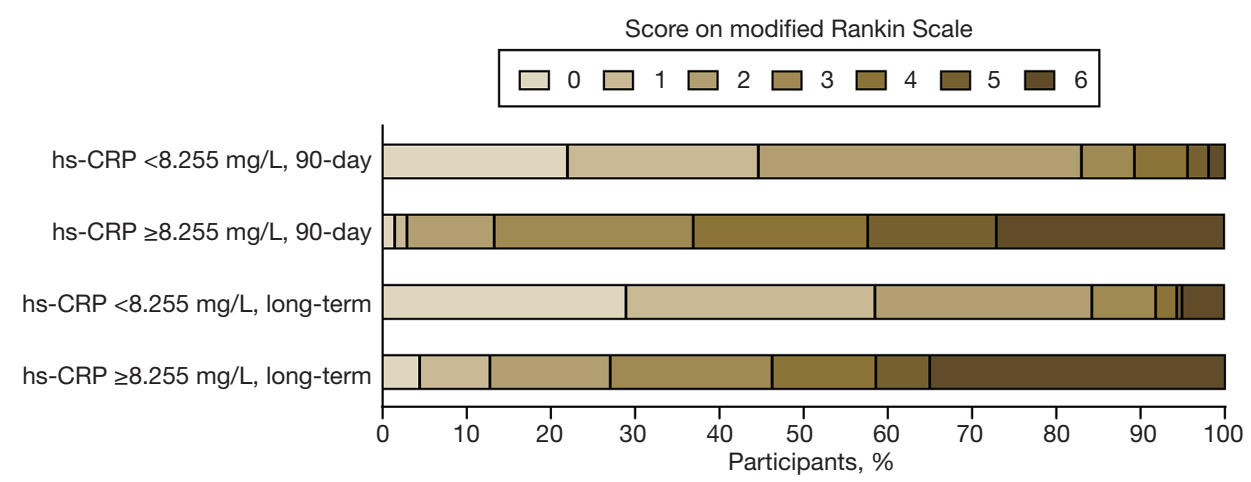

Figure 3 Distribution of the mRS scores at 90-day and long-term follow-up. The percentages are shown in each cell according to the distribution of the mRS scores. Significant differences were observed in the distribution of the mRS scores between patients with a hsCRP level $<8.255 \mathrm{mg} / \mathrm{L}$ and patients with a hs-CRP level $\geq 8.255 \mathrm{mg} / \mathrm{L}$ at both 90 -day $(\mathrm{P}<0.001)$ and long-term follow-up $(\mathrm{P}<0.001)$. $\mathrm{mRS}$, modified Rankin Scale; hs-CRP, high-sensitivity C-reactive protein.

the mRS categories are presented in Table 3 and Figure 3.

Furthermore, Kaplan-Meier curve analysis showed that there was a significant difference between the two groups in long-term survival probability (log-rank, $\mathrm{P}<0.001$; Figure 4), indicating that patients with a hs-CRP level $<8.255 \mathrm{mg} / \mathrm{L}$ have a better long-term survival probability.

\section{Discussion}

In the present study, we investigated the association between hs-CRP levels and clinical outcomes in AIS patients treated with EVT. We found that hs-CRP levels were independently associated with 90-day favorable outcomes.
Patients with a hs-CRP level $<8.255 \mathrm{mg} / \mathrm{L}$ had better functional outcomes at 90-day and long-term follow-up and a higher long-term survival probability than those with an hs-CRP level $\geq 8.255 \mathrm{mg} / \mathrm{L}$. This is the first study to show that hs-CRP levels are associated with clinical outcomes among anterior circulation stroke patients receiving EVT.

Hs-CRP is a sensitive marker of inflammation in the arterial wall (12). Increased hs-CRP levels can reflect the pre-existing situation of the presence of vascular risk factors, as it is related to inflammation of vascular stenosis or occlusion and tissue injury (23). Cerebral ischemic injury can trigger inflammatory processes that evoke acute-phase proteins and inflammatory cytokines (24), and patients with 


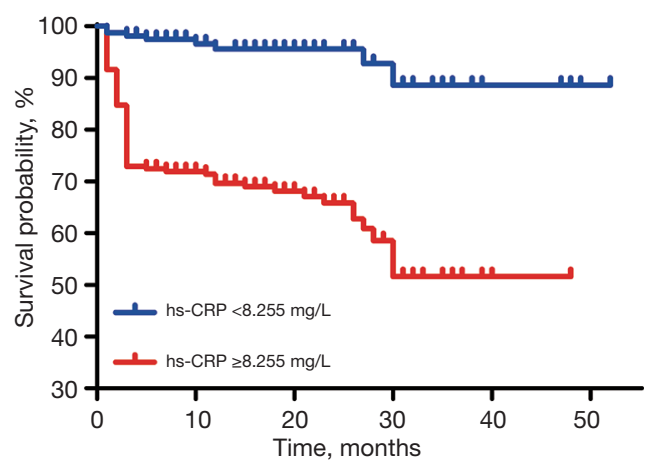

Figure 4 Kaplan-Meier curves for the long-term survival probability. hs-CRP, high-sensitivity C-reactive protein.

larger infarct volumes have a greater neurological deficit and worse functional outcomes (25). Thus, the hs-CRP concentration can, in part, reflect the severity of stroke.

In the present study, elevated hs-CRP levels were associated with poor prognosis at 90-day and long-term follow-up. In line with our findings, the Circulatory Risk in Communities Study (CIRCS) demonstrated a positive association between hs-CRP levels and ischemic stroke in middle-aged Japanese men and women (26). Our findings also corroborate a previous study suggesting that hs-CRP is independently predictive of all-cause mortality (23). However, in the Northern Manhattan Study (NOMAS) (27), a hs-CRP level $>3 \mathrm{mg} / \mathrm{L}$ was associated with ischemic stroke risk in the model adjusted for demographics, but these effects did not persist after controlling for other potential risk factors. The absence of an association with stroke may reflect the heavy risk factor burden; the prevalence of diabetes mellitus and hypertension in the NOMAS vs. our study was $21.5 \%$ vs. $21.3 \%$ and $74.6 \%$ vs. $62.2 \%$, respectively. Other studies similarly found a difference in effect based on the presence or absence of other risk factors. In the Honolulu Heart Study (28), hs-CRP was not associated with stroke among men with hypertension or diabetes or among those aged $>55$ years. Previous studies have demonstrated that advanced age, hypertension, obesity, diabetes, total cholesterol levels, and lower high-density lipoprotein cholesterol (HDL-C) levels were associated with elevated hs-CRP levels $(12,27)$.

In this study, we investigated AIS patients with LVO in the anterior circulation. A previous study demonstrated that hs-CRP levels were elevated in all TOAST (the Trial of Org 10172 in Acute Stroke Treatment) subtypes (29), especially in patients with large artery atherosclerosis.
Rajeshwar et al. (30) found no significant association between hs-CRP levels and the prognosis of patients with lacunar infarcts after 3 months, but in patients with LVO, the association was positive. This was probably because LVO was associated with a larger infarct volume and stronger activation of inflammation than small artery occlusion, which was also associated with changes in hsCRP.

Although the pathophysiological association between hs-CRP and LVO is unclear, different mechanisms may be involved. Hs-CRP is related to focal inflammation, and the inflammatory response plays an important role in patients with LVO, as it is associated with stenosis or occlusion of intracranial or extracranial large arteries, unstable plaque, artery-to-artery embolism and hypoperfusion (31). On the other hand, hs-CRP can bind to the plasma membranes and small nuclear ribonucleoprotein particles in the exposed nuclei of damaged cells (32). After such binding, the classical complement pathway can be activated, which may therefore contribute to ischemic tissue damage. A combination of these mechanisms may better explain the association between hs-CRP and LVO. Further experiments are needed to confirm the exact mechanism of prognosis.

Furthermore, the hs-CRP measurement time point at which clinical outcomes are most emblematic has not been established. In this study, the hs-CRP concentration was measured immediately upon admission. The serum hsCRP concentration increases early and remains elevated above control values at 3 months after the index stroke (33), but to what extent the elevation reflects the inflammatory response to stroke is unclear. Previous studies suggested the usefulness of a sample taken within 12 to 72 hours after stroke onset (18,34,35). Di Napoli et al. (36) measured serum hs-CRP within 24 hours after index ischemic stroke, within 48 to 72 hours, and at hospital discharge; they found that hs-CRP at hospital discharge was the strongest independent marker of adverse outcome (hazard ratio, 7.42; 95\% CI, 2.75-20.03; $\mathrm{P}=0.0001)$, whereas further investigation suggested that the peak measurement in the first week after stroke onset was particularly valuable in relation to infarct volume and clinical outcomes (33). It is possible that discharge concentrations more closely reflect the baseline inflammatory status of the patients, and thus their intrinsic risk caused by inflammatory activity. Further prospective investigations of the optimal timing of hs-CRP for use as a prognostic marker after AIS with EVT are warranted.

This study has certain limitations. The nonrandomized 
single-center design of our study had limitations in nature, and the sample population cannot represent the general population of AIS with EVT. In view of the changes in the time window of EVT for AIS patients in recent years, the median time from stroke onset to recanalization in this study has exceeded 8 hours, which is inconsistent with the traditional 8-hour time window in most previous studies. Therefore, care should be taken in generalizing these results to settings where time to recanalization is shorter, and future studies are needed to further verify our conclusions. Moreover, the timing of plasma sampling concerning stroke onset may have influenced our findings; thus, serial hs-CRP measurements should be performed. As dynamic change measurement of hs-CRP may provide more prognostic relevance in AIS, standardized serial measurements of hsCRP concentration should be the focus in future studies to determine whether dynamic changes are related to prognosis. Finally, although multivariable logistic regression analysis was performed to adjust for relevant variables, unmeasured and residual confounding factors remained.

\section{Conclusions}

Our present study demonstrated that hs-CRP levels were associated with clinical outcomes of AIS patients undergoing EVT with LVO in the anterior circulation. These results need to be further verified in larger multicenter prospective studies.

\section{Acknowledgments}

Funding: This study was supported by Cheung Kong (Chang Jiang) Scholars Program (T2014251), National Key Research and Development Program of China (2016YFC1301502), and National Natural Science Foundation of China (81771260, 81601006, 81620108011).

\section{Footnote}

Reporting Checklist: The authors have completed the STROBE reporting checklist. Available at http://dx.doi. org/10.21037/atm-20-3820

Data Sharing Statement: Available at http://dx.doi. org/10.21037/atm-20-3820

Conflicts of Interest: All authors have completed the ICMJE uniform disclosure form (available at http://dx.doi. org/10.21037/atm-20-3820). The authors have no conflicts of interest to declare.

Ethical Statement: The authors are accountable for all aspects of the work in ensuring that questions related to the accuracy or integrity of any part of the work are appropriately investigated and resolved. The study was conducted in accordance with the Declaration of Helsinki (as revised in 2013). The study was approved by the Ethics Committee of Xuanwu Hospital (No. 2017030), and informed consent was taken from all the patients.

Open Access Statement: This is an Open Access article distributed in accordance with the Creative Commons Attribution-NonCommercial-NoDerivs 4.0 International License (CC BY-NC-ND 4.0), which permits the noncommercial replication and distribution of the article with the strict proviso that no changes or edits are made and the original work is properly cited (including links to both the formal publication through the relevant DOI and the license). See: https://creativecommons.org/licenses/by-nc-nd/4.0/.

\section{References}

1. GBD 2017 Causes of Death Collaborators. Global, regional, and national age-sex-specific mortality for 282 causes of death in 195 countries and territories, 1980-2017: a systematic analysis for the Global Burden of Disease Study 2017. Lancet 2018;392:1736-88.

2. Feigin VL, Roth GA, Naghavi M, et al. Global burden of stroke and risk factors in 188 countries, during 1990-2013: a systematic analysis for the Global Burden of Disease Study 2013. Lancet Neurol 2016;15:913-24.

3. Feigin VL, Nguyen G, Cercy K, et al. Global, regional, and country-specific lifetime risks of stroke, 1990 and 2016. N Engl J Med 2018;379:2429-37.

4. Powers WJ, Rabinstein AA, Ackerson T, et al. Guidelines for the early management of patients with acute ischemic stroke: 2019 update to the 2018 guidelines for the early management of acute ischemic stroke: a guideline for healthcare professionals from the American Heart Association/American Stroke Association. Stroke 2019;50:e344-418.

5. Grech R, Pullicino R, Thornton J, et al. An efficacy and safety comparison between different stentriever designs in acute ischaemic stroke: a systematic review and metaanalysis. Clin Radiol 2016;71:48-57.

6. Goyal M, Menon BK, van Zwam WH, et al. Endovascular 
thrombectomy after large-vessel ischaemic stroke: a metaanalysis of individual patient data from five randomised trials. Lancet 2016;387:1723-31.

7. Bouslama M, Haussen DC, Aghaebrahim A, et al. Predictors of good outcome after endovascular therapy for vertebrobasilar occlusion stroke. Stroke 2017;48:3252-7.

8. Shi ZS, Liebeskind DS, Xiang B, et al. Predictors of functional dependence despite successful revascularization in large-vessel occlusion strokes. Stroke 2014;45:1977-84.

9. Yoon W, Kim SK, Park MS, et al. Predictive factors for good outcome and mortality after stent-retriever thrombectomy in patients with acute anterior circulation stroke. J Stroke 2017;19:97-103.

10. Danton GH, Dietrich WD. Inflammatory mechanisms after ischemia and stroke. J Neuropathol Exp Neurol 2003;62:127-36.

11. Du Clos TW, Mold C. C-reactive protein: an activator of innate immunity and a modulator of adaptive immunity. Immunol Res 2004;30:261-77.

12. Pearson TA, Mensah GA, Alexander RW, et al. Markers of inflammation and cardiovascular disease: application to clinical and public health practice: a statement for healthcare professionals from the Centers for Disease Control and Prevention and the American Heart Association. Circulation 2003;107:499-511.

13. VanGilder RL, Davidov DM, Stinehart KR, et al. C-reactive protein and long-term ischemic stroke prognosis. J Clin Neurosci 2014;21:547-53.

14. Cai Z, He W, Zhuang FJ, et al. The role of high highsensitivity C-reactive protein levels at admission on poor prognosis after acute ischemic stroke. Int J Neurosci 2019;129:423-9.

15. Qiu R, Gao Y, Hou D, et al. Association between hsCRP levels and the outcomes of patients with small-artery occlusion. Front Aging Neurosci 2016;8:191.

16. Wang L, Jiang J, Du L, et al. The prognostic value of serum pregnancy-associated plasma protein A, S100 and high-sensitivity C-reactive protein in acute ischemic stroke patients without heparin administration. Clin Biochem 2014;47:187-91.

17. Song IU, Kim JS, Kim YI, et al. Relationship between high-sensitivity $\mathrm{C}$-reactive protein and clinical functional outcome after acute ischemic stroke in a Korean population. Cerebrovasc Dis 2009;28:545-50.

18. den Hertog HM, van Rossum JA, van der Worp HB, et al. C-reactive protein in the very early phase of acute ischemic stroke: association with poor outcome and death. J Neurol 2009;256:2003-8.
19. von Elm E, Altman DG, Egger M, et al. The Strengthening the Reporting of Observational Studies in Epidemiology (STROBE) statement: guidelines for reporting observational studies. Lancet 2007;370:1453-7.

20. Brott T, Adams HP Jr, Olinger CP, et al. Measurements of acute cerebral infarction: a clinical examination scale. Stroke 1989;20:864-70.

21. Barber PA, Demchuk AM, Zhang J, et al. Validity and reliability of a quantitative computed tomography score in predicting outcome of hyperacute stroke before thrombolytic therapy. ASPECTS Study Group. Alberta Stroke Programme Early CT Score. Lancet 2000;355:1670-4.

22. Bonita R, Beaglehole R. Recovery of motor function after stroke. Stroke 1988;19:1497-500.

23. Itrat A, Griffith SD, Alam S, et al. The role of very high high-sensitivity C-reactive protein levels on mortality after stroke. J Neurol Sci 2017;372:1-5.

24. Gill D, Veltkamp R. Dynamics of T cell responses after stroke. Curr Opin Pharmacol 2016;26:26-32.

25. Beloosesky Y, Streifler JY, Burstin A, et al. The importance of brain infarct size and location in predicting outcome after stroke. Age Ageing 1995;24:515-8.

26. Chei CL, Yamagishi K, Kitamura A, et al. C-reactive protein levels and risk of stroke and its subtype in Japanese: the Circulatory Risk in Communities Study (CIRCS). Atherosclerosis 2011;217:187-93.

27. Elkind MS, Luna JM, Moon YP, et al. High-sensitivity C-reactive protein predicts mortality but not stroke: the Northern Manhattan Study. Neurology 2009;73:1300-7.

28. Curb JD, Abbott RD, Rodriguez BL, et al. C-reactive protein and the future risk of thromboembolic stroke in healthy men. Circulation 2003;107:2016-20.

29. Matsuo R, Ago T, Hata J, et al. Plasma C-reactive protein and clinical outcomes after acute ischemic stroke: a prospective observational study. PLoS One 2016;11:e0156790.

30. Rajeshwar K, Kaul S, Al-Hazzani A, et al. C-reactive protein and nitric oxide levels in ischemic stroke and its subtypes: correlation with clinical outcome. Inflammation 2012;35:978-84.

31. Zeng L, He X, Liu J, et al. Differences of circulating inflammatory markers between large- and small vessel disease in patients with acute ischemic stroke. Int J Med Sci 2013;10:1399-405.

32. Gill R, Kemp JA, Sabin C, et al. Human C-reactive protein increases cerebral infarct size after middle cerebral artery occlusion in adult rats. J Cereb Blood Flow Metab 
2004;24:1214-8.

33. Emsley HC, Smith CJ, Gavin CM, et al. An early and sustained peripheral inflammatory response in acute ischaemic stroke: relationships with infection and atherosclerosis. J Neuroimmunol 2003;139:93-101.

34. Christensen H, Boysen G. C-reactive protein and white blood cell count increases in the first 24 hours after acute stroke. Cerebrovasc Dis 2004;18:214-9.

Cite this article as: Wang $\mathrm{L}, \mathrm{Wu} \mathrm{L}$, Lang $\mathrm{Y}, \mathrm{Wu} \mathrm{D}$, Chen J, Zhao W, Li C, Ji X. Association between high-sensitivity C-reactive protein levels and clinical outcomes in acute ischemic stroke patients treated with endovascular therapy. Ann Transl Med 2020;8(21):1379. doi: 10.21037/atm-20-3820
35. Montaner J, Fernandez-Cadenas I, Molina CA, et al. Poststroke C-reactive protein is a powerful prognostic tool among candidates for thrombolysis. Stroke 2006;37:1205-10.

36. Di Napoli M, Papa F, Bocola V. C-reactive protein in ischemic stroke: an independent prognostic factor. Stroke 2001;32:917-24. 